\title{
Design of Ground Control Station for Operation of Multiple Combat Entities
}

\author{
Jinyeong Heo', Suhwan Kim², Yongjin James Kwon ${ }^{*}$ \\ ${ }^{1}$ Department of Industrial Engineering, Ajou University, Suwon, South Korea \\ ${ }^{2} 1^{\text {st }}$ Division, $3^{\text {rd }}$ Department, Agency for Defense Development, Daejeon, South Korea \\ Email: *yk73@ajou.ac.kr
}

Received 3 May 2016; accepted 19 May 2016; published 26 May 2016

\begin{abstract}
Recently, if you look at the trend of the Unmanned Combat Entities (UCE) on the world that are actually operational, a large number of personnel per one UCE has been operating the GCS. However, UCEs to perform the attack/reconnaissance mission are very expensive assets and require a considerable amount of time to train for UCE operations. Accordingly, the future battlefield environment has become important to develop multiple UCE ground control station. In this study, we developed a multiple UCE GCS that one operator can operate up to four UCEs. The software was built with a total of 6 displays using a Lockheed Martin Corporation's prepar3D. Scenario of research takes into account the operation of the South Korea-type future multiple UCEs, to take advantage of the simulation system, in this paper, we propose for each of the concepts and technologies.
\end{abstract}

\section{Keywords}

Ground Control Station, Multiple Unmanned Combat Entities, Mission Planning System, Ergronomics Design, Task Load

\section{Introduction}

Under future battle environment, UCEs will be utilized as a key element for battlefield in that it will be conducting missions such as striking enemy's core forces by receiving information on UCE's location and status, and transmitting control orders to the UCEs through real-time battlefield data collection and sharing. Recently, there have been active researches conducted on developing the technology from single UCE, in which one operator exists for one corresponding UCE, towards multiple UCE system, in which one person operates multiple UCEs [1]. Therefore, there are a need for a ground control system that can effectively control diverse multiple UCEs and conduct missions, and for the human interface that can minimize mission assignments for the operators [2]. Considering such issues, we have established a UCE GCS station which can integrate and control up to 4 UCEs that used to be independently employed. Figure 1 is the overall structure of the GCS station we established, and for the detailed factors, there are front image, infrared image display, and for the parts that operators

"Corresponding author. 


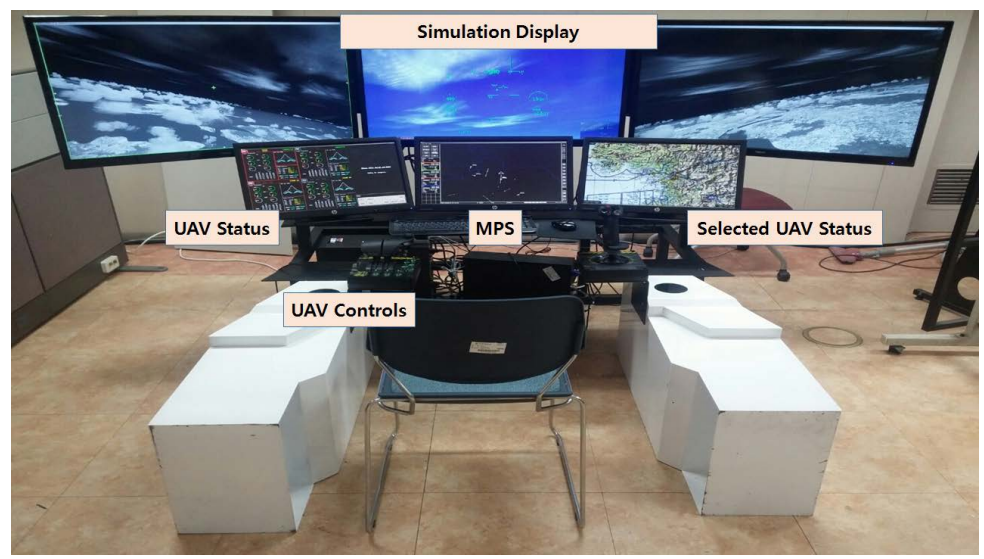

Figure 1. Multi-UCE GCS entire structure.

use, there are UCE selection, status display, activation display, mission plan display and selected UCE information display. In this research, we will define the overall structure of the hardware of the GCS station that has been invented, and secondly suggest detailed technology for the software and the concepts of employment.

\section{Design of Hardware and Software}

\subsection{Hardware Structure}

Figure 2 is simply to show the structure of the ground control station implemented in this study. Large display in front of the operator can see the front image and the infrared image of the UCE. In addition, three displays in front of the large display is operated in a touch screen method and are able to grasp information UCE, they are possible to operate by using a UCE Control.

\subsection{Mission Planning System and the Design of Display}

Mission plan system is a system that facilitates on-ground operators to quickly conduct missions with sufficiently prearranged plans including flight path, time, fuel, arms, tactics, action manual and map. In other words, it functions as a system to prepare and plan for various issues needed for self-regulated flight operation of UCEs, and the mission plan system organization that has been designed for this research is based on the organization diagram in Figure 3.

Firstly, before designing mission plan system, we have conducted the Mission Generator technical research. It is composed of three modules: Body Behavior Generator that generates paths for UCEs, Effector Behavior Generator that generates the behaviors of Effector which is carried in the body when behavior information such as the angle of the body is determined, and lastly, Mission Binder which links previous two modules and makes sure they operate properly. It generates the behaviors of Body and Effector based on the data provided from Database, and the behaviors that are generated will generate the final mission considering environmental factors through Mission Binder. Secondly, utilizing the data from GIS (Geographic Information System), it has established technology to set restricted air zone, designate Way-point for missions and design events, and set up a database by transition and application of 2D-3D geographic information data. Lastly, we can categorize the MPS display composition principle into standardization principle and design differentiation principle, and in this research, we will be following the standardization criterion, as Figure $\mathbf{4}$ shown below, for ergonomic design/tactics object expression/GCS design.

Figure 5 is the basic display designed through the MPS display composition principle which is mentioned above. This display is composed of mission information, data of each UCE, and other user-selected information. Basically, the operator can grasp the information of four UCEs at a glance, selectively show or take away information that the user needs such as electronic map filters, and it shows real-time information such as location, velocity and orientation of the UCE on MPS Digital Map. In addition, the display can be represented by selecting information. For example, Topographic Information, ORB, Vehicle Identifier, Instrument Landing System, Flight Information Region and so on. Figure 6 and Figure 7 show a screen showing optional information. 


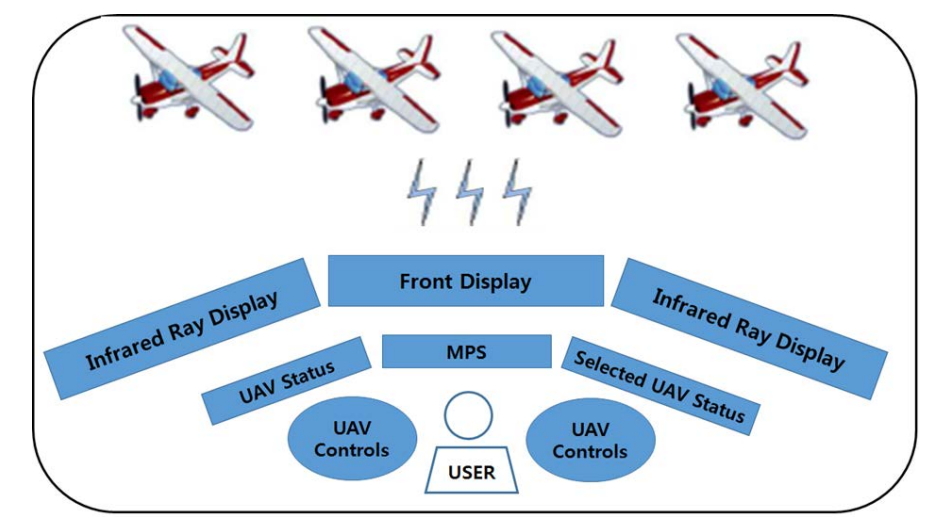

Figure 2. Multi-UCE GCS.

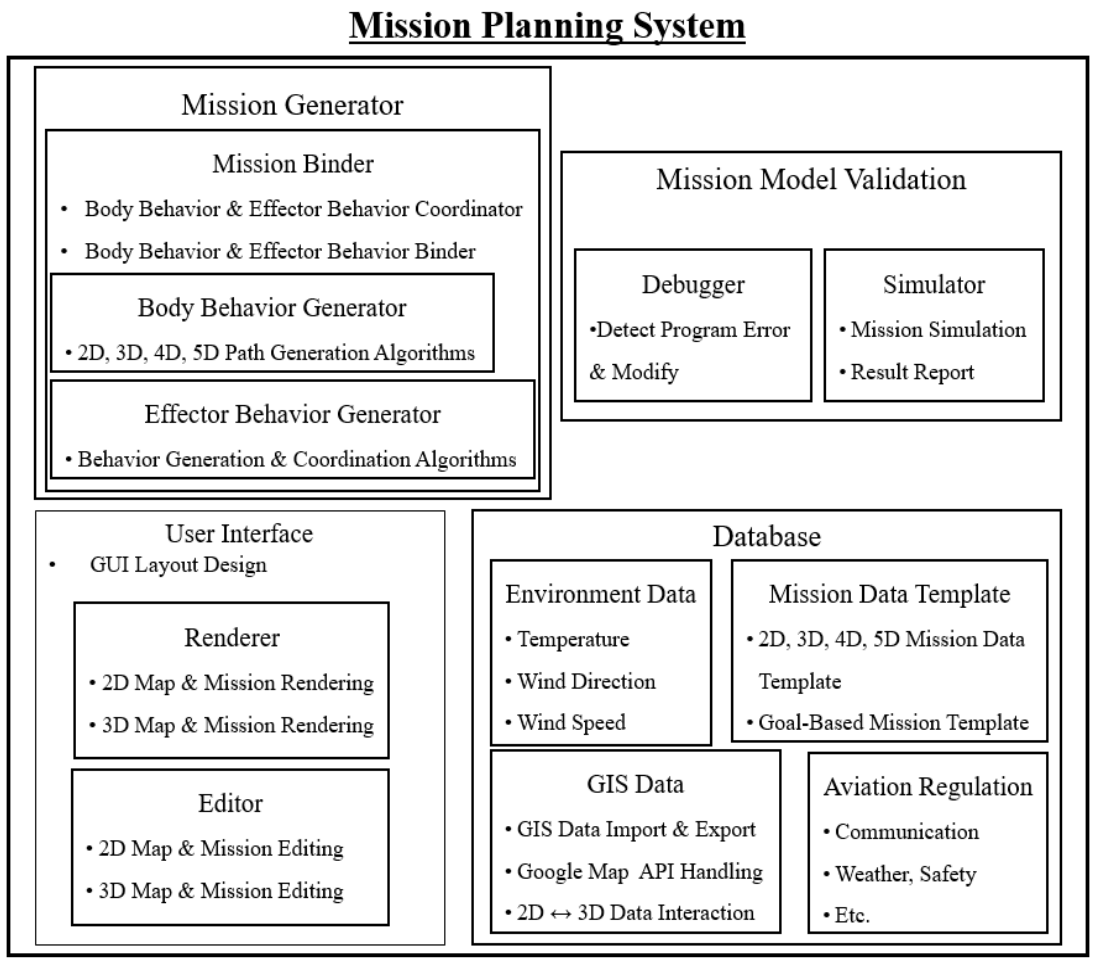

Figure 3. Mission planning system diagram.

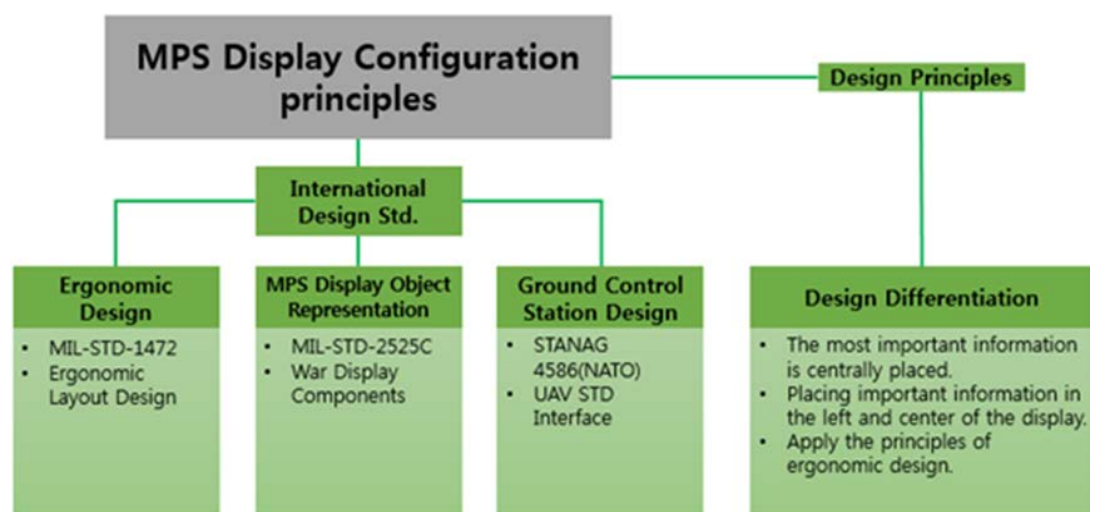

Figure 4. MPS display conceptual configuration principles. 


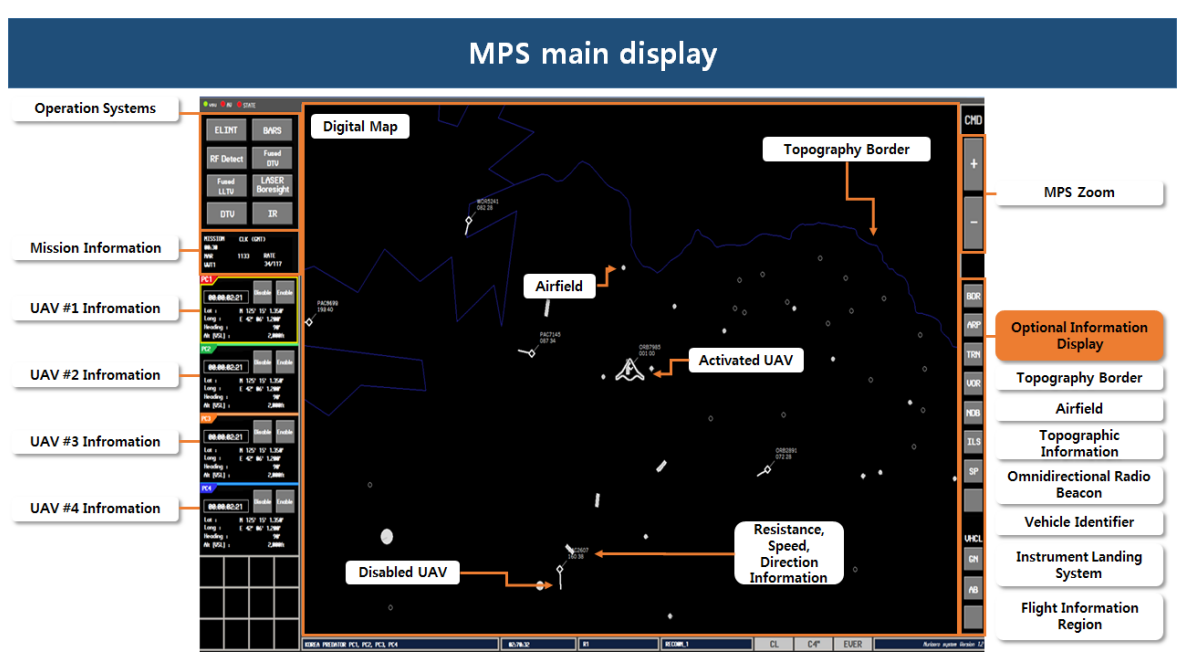

Figure 5. MPS main display.

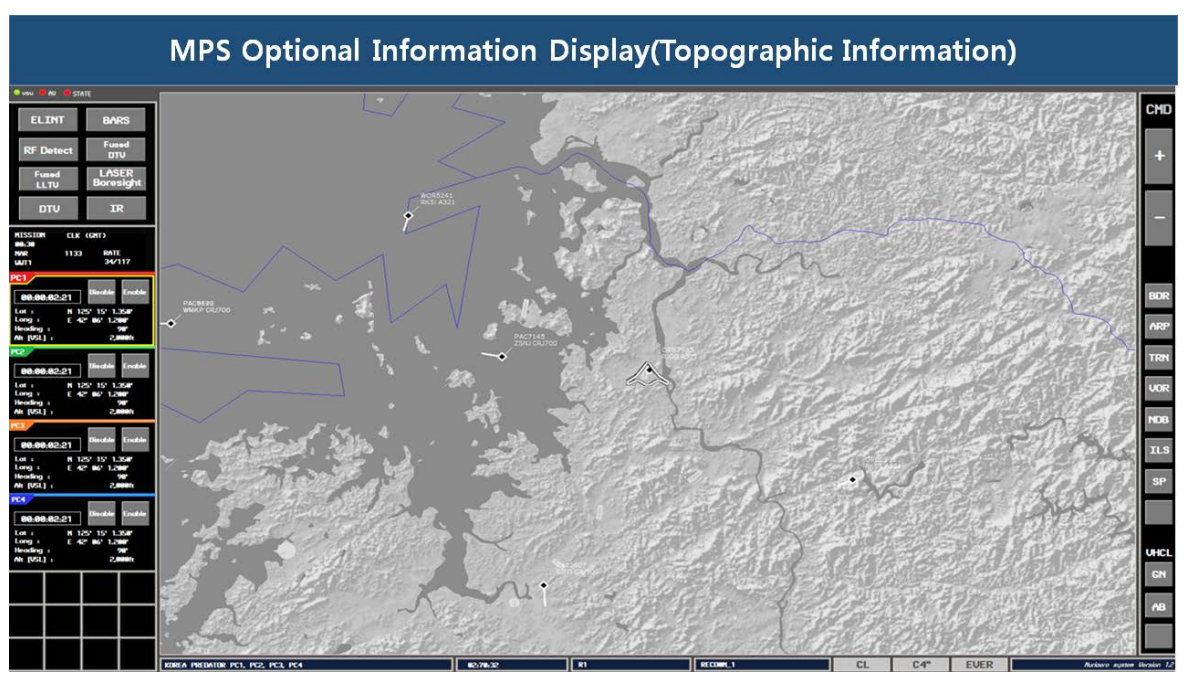

Figure 6. MPS user optional information display example (Topographic Information).

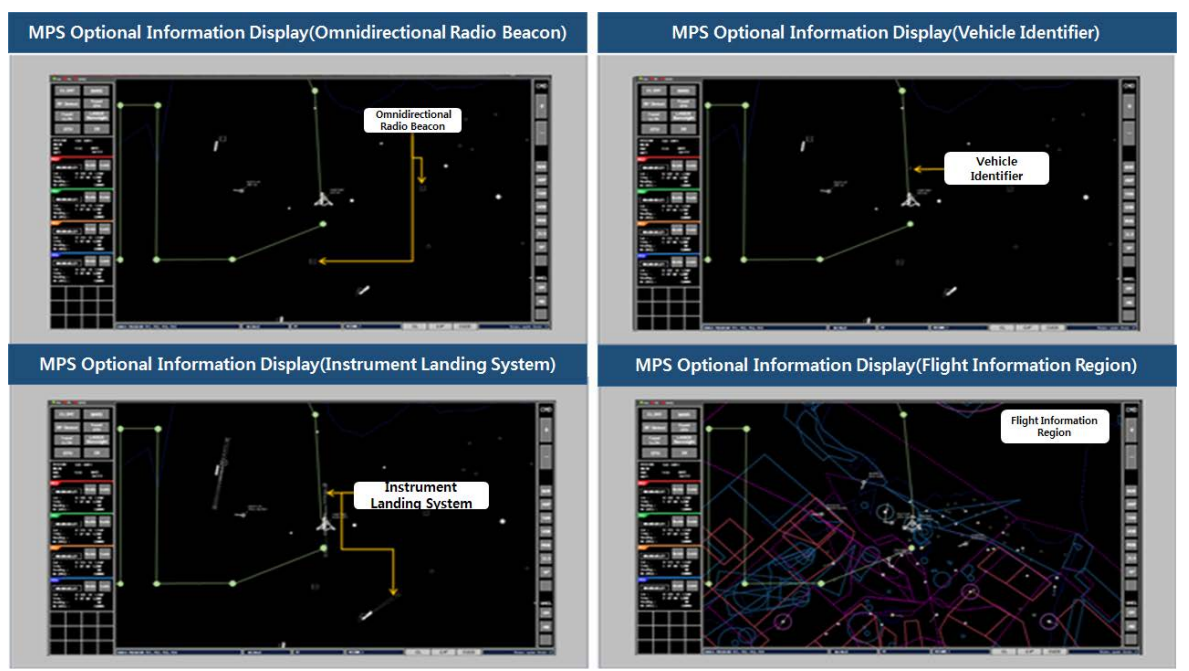

Figure 7. Example (ORB, Vehicle Identifier, Instrument Landing System, Flight Information Region). 


\subsection{UCE Status Information}

Figure 8 shows status information for up to four UCEs, and enables the operators to select the UCE that he/she is willing to activate and see the status of each UCE at a glance. We can employ the UCEs appropriately for the given mission conditions by selecting operational modes such as air-to-air, air-to-ground or tactical reconnaissance, and the activated UCE can enhance the mission effectiveness by letting the operator select information for the extra information display. The extra information display's composition is as shown in Figure 9, and we can check information including meter, radar, arm status, oil pressure and power.

\subsection{Selected UCE Information}

In selected UCE information display (Figure 10), it shows the actual location on the map where the selected UCE is flying at the moment. This can be zoomed in and out, and has advantages to efficiently utilize information such as geographic, flight and tactical data.

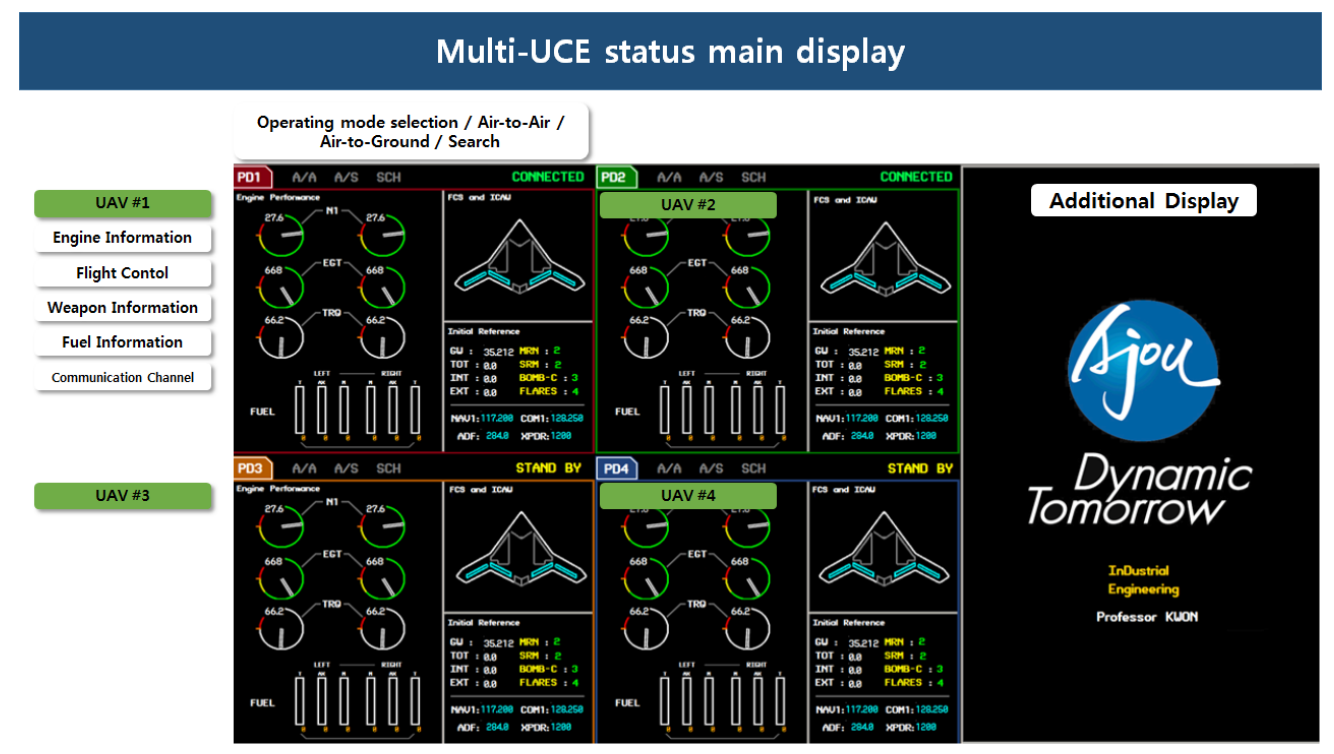

Figure 8. Multi-UCE status display example (main display).

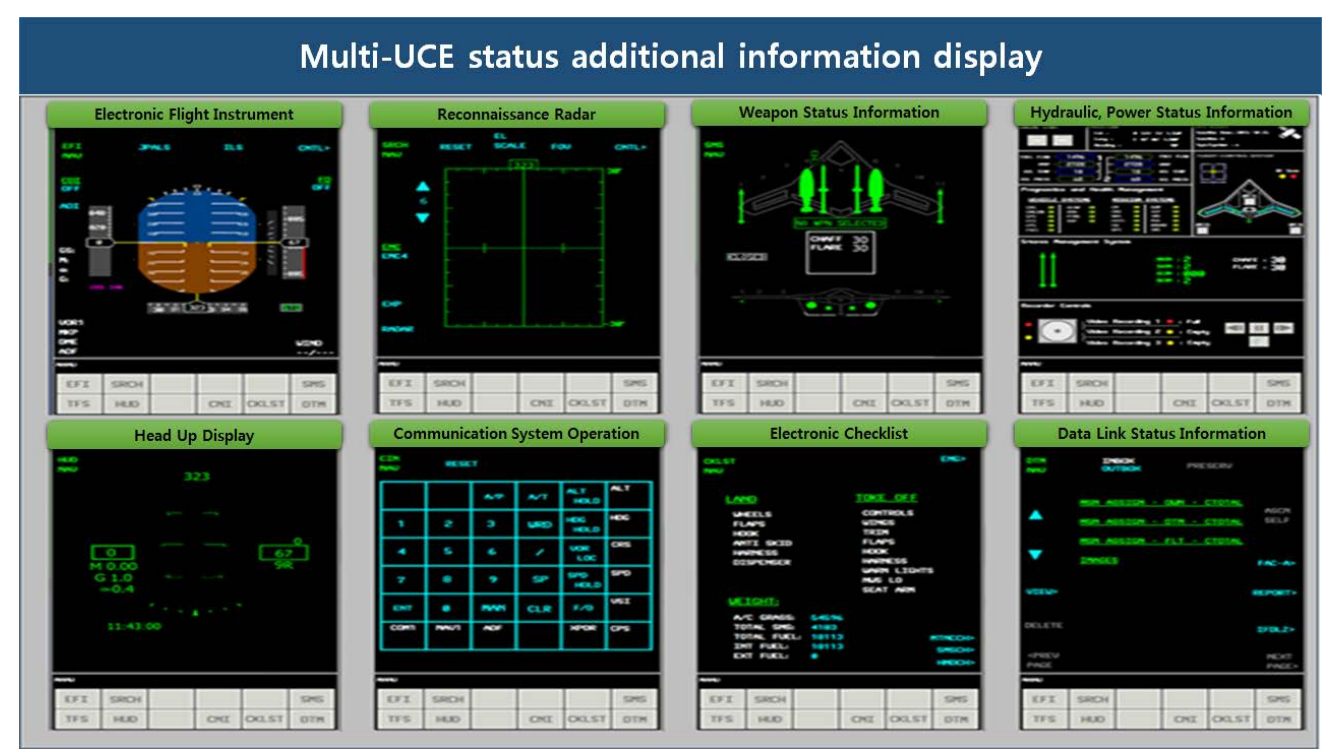

Figure 9. Multi-UCE status display example (additional display) main display. 


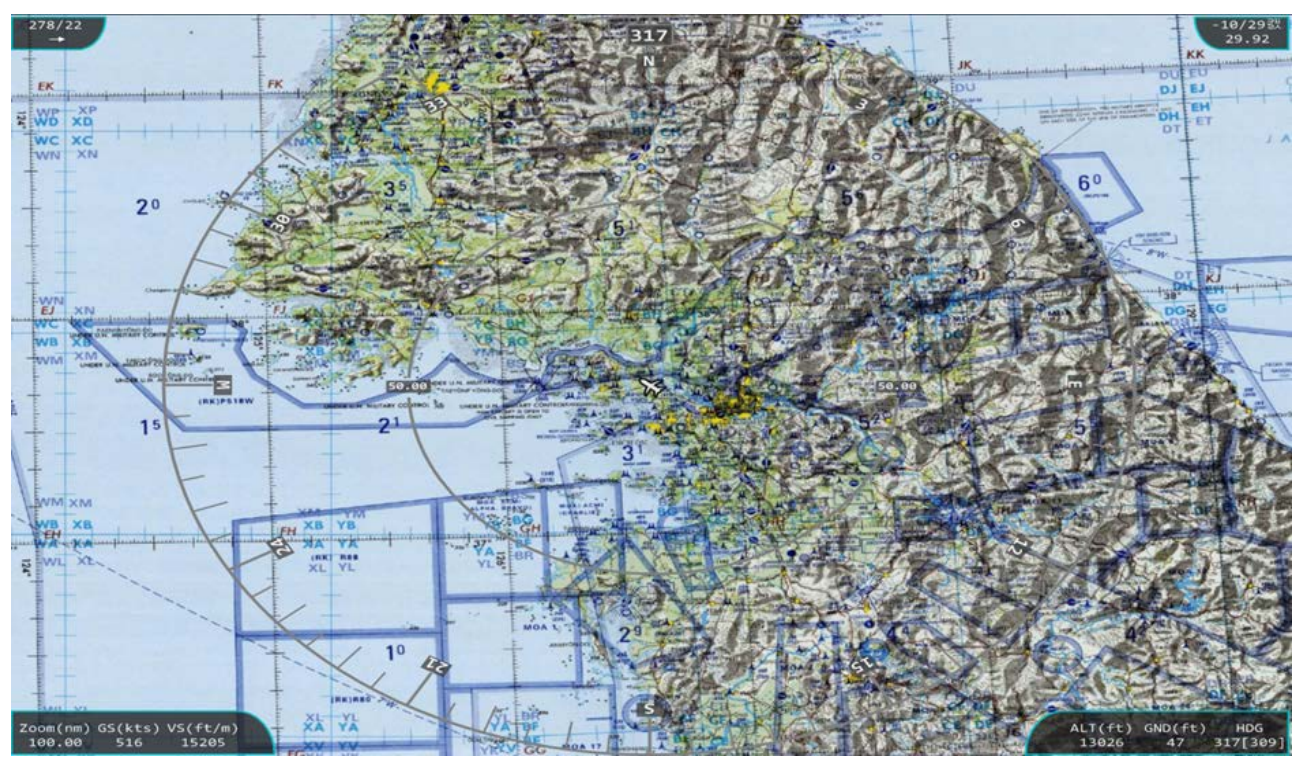

Figure 10. Selected UCE moving map information.

\section{Conclusion}

In this research, by designing a ground control station that controls up to 4 UCEs, we have invented a multiple UCE GCS, which enables us to more effectively conduct missions regarding the employment of multiple UCEs. Since the ground control software design, through information display of multiple UCEs, can let the operators choose a certain UCE that he/she wants to activate, grasp the status of each UCE at a glance, operate the UCEs appropriately for mission conditions by assigning employment modes to each UCE such as air-to-air, air-toground and tactical reconnaissance, and let the operator select information to display for the extra information display, we expect the mission effectiveness to be raised through this.

\section{Acknowledgements}

This work was supported by the Agency for Defense Development (ADD) under the Contract No. UD140066CD. The authors wish to express sincere gratitude for the financial support.

\section{References}

[1] Shin, Y.H., Cho, S.W., Jo, S.B., Kim, S.H., Lyu, C.K. and Choi, K. (2012) Development of Ground Control Software for Operation of Multiple Unmanned Aerial Vehicles. Journal of The Korean Society for Aeronautical and Space Sciences, 40, 542-547. http://dx.doi.org/10.5139/JKSAS.2012.40.6.542

[2] Ryu, C.H. and Ra, S.W. (2005) Mission Planning and Control Analysis Algorithms for the Safe Flight of an Unmanned Aerial Vehicle. Journal of The Korean Society for Aeronautical and Space Sciences, 33, 71-77.

http://www.dbpia.co.kr/Article/NODE01102209

http://dx.doi.org/10.5139/JKSAS.2005.33.3.071 